\title{
Numerically Exact Analysis of a Two-Dimensional Variable-Resistivity Reflector Fed by a Complex-Point Source
}

\author{
Alexander I. Nosich, Senior Member, IEEE, Vladimir B. Yurchenko, and Ayhan Altintaş, Senior Member, IEEE
}

\begin{abstract}
Accurate numerical analysis of a two-dimensional (2-D) variable-resistivity reflector has been carried out by the method of regularization based on the analytical inversion of the corresponding static problem. The complex source-point model has been used to account for the directivity of the feeder and both the $\mathrm{H}$ - and E-polarization cases are considered. Far-field radiation patterns, directivity, and total radiative power have been computed for reflectors of uniform and nonuniform complex resistivities. The concept of edge loading for the control and improvement of antenna characteristics is confirmed by this numerically rigorous technique.
\end{abstract}

Index Terms-Moment methods, reflector antennas.

\section{INTRODUCTION}

$\mathbf{I}$ T was probably in the early 1970's that lowering the sidelobe level of a reflector antenna by means of imperfect rim loading was first discussed [1]. The physical idea that underlines this advice is quite clear: a perfectly conducting sharp edge of a reflector is too strong a scatterer for a feed field, even if a reasonable edge illumination taper is used. To have it better matched with the free-space, it can be suggested to make the reflector's rim slightly transparent or absorbing. Probably the best theoretical study of this idea was presented in [2] and [3], where the geometrical theory of diffraction (GTD) corrected for the main-beam direction by physical optics (PO) was used to simulate the beamforming by a parabolic reflector with a loaded rim. However, both PO and GTD are high-frequency techniques that do not have a uniform accuracy. Therefore, a comparison with a more accurate integral-equation (IE)-based analysis can be interesting. Besides, in [2] and [3] the loading was assumed as an impedance nontransparent (e.g., coated) rim, while a partially penetrable one is also of interest. Theoretically, the transparent or absorbing nature of a thin-reflector material may be simulated through the concept of resistivity, attributed to

Manuscript received August 6, 1996; revised March 28, 1997. This work was supported in part by the Scientific and Technical Research Council of Turkey (TUBITAK).

A. I. Nosich is with the Institute of Radiophysics and Electronics, National Academy of Sciences of Ukraine, Kharkov, 310085 Ukraine.

V. B. Yurchenko is with the Institute of Radiophysics and Electronics, National Academy of Sciences of Ukraine, Kharkov, 310085 Ukraine. He is also with the Department of Electrical and Electronics Engineering, Bilkent University, Bilkent, Ankara, 06533 Turkey.

A. Altintaş is with the Department of Electrical and Electronics Engineering, Bilkent University, Bilkent, Ankara, 06533 Turkey.

Publisher Item Identifier S 0018-926X(97)08002-2. the rim area. This means that the surface IE approach should be modified to incorporate thin imperfect curved scatterers of variable resistivity.

Another consideration that justifies a revisiting of the resistive reflector analysis is the presence of commercial Ku-band reflectors made of a fiberglass layer bonded to a thin metal screen. In fact, modern printed technologies are able to produce thin resistive layers of several hundred ohms per square. Using such reflectors deposited on a lightweight substrate can be a promising technology for spaceborn, manpack, and other antenna applications.

In the present paper, we extend our previous analysis of perfect electric conducting (PEC) two-dimensional (2-D) reflector antennas [4] to variable-resistivity reflectors. The latter can be either uniformly resistive (like a thin dielectric surface) or have only the edge area that is partially transparent and/or absorbing. We use the method-of-regularization (MoR) approach, which can be considered as a sophisticated version of method of moments (MoM) due to a judicious choice of expansion functions in the form of an orthogonal set of eigenfunctions of the static part of the IE kernel. This approach implies analytical inversion of the extracted part of the IE that enables a numerical solution of the "rest" in a very efficient and rapidly convergent manner. Mathematically, it means that when discretizing the IE we transform it to a Fredholm secondkind matrix equation, instead of one of the first kind as with the conventional MoM. The gain of such a sophistication is that electrically much larger scatterers can be computed with desktop hardware and the accuracy is uniformly guaranteed. To account for the directive character of the feed field but to avoid inaccuracies caused by its approximate description, we take it as a complex-source field, which is an exact solution the Helmholtz equation. Thus, our analysis is numerically to rigorous and the results can be considered as reference data. Although it does not enable one to study the crosspolarization and aperture-blockage effects, it fully incorporates such mechanisms as surface curvature, power loss, and guided and creeping wave contributions.

The assumed time dependence $e^{-i \omega t}$ is omitted throughout the analysis.

\section{PROBlem Formulation}

The problem of the wave scattering from a resistive reflector is formulated similarly to that for a PEC reflector [4]. The total 
field is characterized by the single scalar function

$$
U=U^{i n}+U^{s c}
$$

which is either $E_{z}$ or $H_{z}$ component depending on the polarization. This function is to solve the Helmholtz equation off the reflector $M$ and satisfy the boundary conditions at $M$, the Sommerfeld radiation condition at infinity, and the edge condition such that the field energy is limited in any finite domain around the edge.

A resistive-surface boundary condition has been introduced as a convenient approximation of a partially transparent (or penetrable) thin material sheet of high intrinsic impedance (see [5]-[8]). It can be written in the form of the following set of surface equations:

$$
\begin{aligned}
\frac{1}{2}\left[\vec{E}_{T}^{+}(\vec{r})+\vec{E}_{T}^{-}(\vec{r})\right] & =R(\vec{r}) \vec{n}(\vec{r}) \times\left[\vec{H}_{T}^{+}(\vec{r})-\vec{H}_{T}^{-}(\vec{r})\right] \\
\vec{E}_{T}^{+}(\vec{r}) & =\vec{E}_{T}^{-}(\vec{r})
\end{aligned}
$$

coupling the limiting values of the tangential field components. The superscripts "-" and "+" are related to the front and rear faces of the reflector, respectively, and the normal $\vec{n}(\vec{r})$ is directed from the "-" to "+" face.

Resistivity $R$ in (2) can be complex-valued and a function of the position on $M$ but is assumed isotropic. Note that the transition to a PEC reflector is arranged by setting $R=0$. The two most frequently encountered examples of a resistive surface are given by the model of a thin dielectric sheet of thickness $h$ and relative permittivity $\epsilon_{r}$ and that of a metal sheet of the conductivity $\sigma$, namely

$$
R_{\text {diel }}=\frac{i Z_{0}}{k h\left(\epsilon_{r}-1\right)}, \quad R_{\text {metal }}=\frac{1}{h \sigma}
$$

where $Z_{0}$ is the free-space impedance. Note that a lossless sheet is obtained if $\operatorname{Re} R=0$. Otherwise, the reflector is lossy and a part of the incident field is absorbed in the reflector material.

In accordance with the above-mentioned desire to have the incident field directive, yet an exact solution of the Helmholtz equation, we take it as a complex-point source field

$$
U^{i n}(\vec{r})=C H_{0}^{(1)}\left(k\left|\vec{r}-\left(\vec{r}_{0}+i \vec{b}\right)\right|\right)
$$

where the parameter $k b$ corresponds to the aperture of an actual feed and $C$ is a real constant. Further, we shall concentrate on the front-fed reflector geometry, so we shall take angle $\vec{b}=0$. Such a source is known to produce a beam of width governed by the value of $k b$ (see [9]). As was pointed out in [4], the directivity $D_{0}$ and the total radiated power $P_{0}$ of this feed in free-space are given by

$$
D_{0}=\frac{e^{2 k b}}{I_{0}(2 k b)}, \quad P_{0}=C^{2} \frac{2 \eta}{k} I_{0}(2 k b)
$$

where $\eta$ is $Z_{0}$ in the $\mathrm{H}$ case and $1 / Z_{0}$ in the $\mathrm{E}$ case, and $I_{0}$ is the modified Bessel function. For large values of $k b, D_{0}$ and $P_{0}$ behave as $(k b)^{1 / 2}$ and $e^{2 k b}(k b)^{-1 / 2}$, respectively.

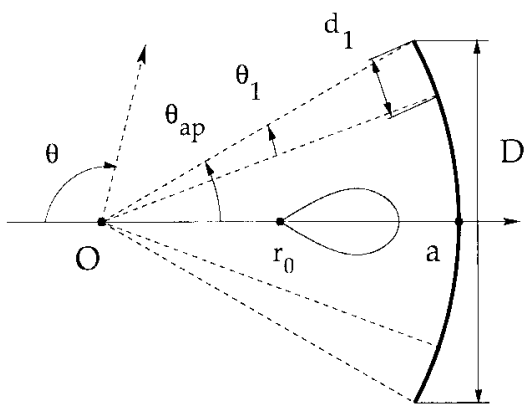

Fig. 1. Two-dimensional reflector geometry $\left[F=a-r_{0}=a / 2\right.$, $\left.D=2 a \sin \left(\theta_{a p}\right)\right]$.

\section{BASIC EQUATIONS FOR THE H-Polarization CASE}

In this case, we denote the $z$-component scattered magnetic field function as $H^{s c}$ and seek it as a double-layer potential given by

$$
H^{s c}(\vec{r})=\int_{M} Y\left(\vec{r}^{\prime}\right) \frac{\partial}{\partial n^{\prime}} G_{0}\left(\vec{r}, \vec{r}^{\prime}\right) d l^{\prime}
$$

where the kernel function is the normal derivative of the free-space Green's function

$$
G_{0}\left(\vec{r}, \vec{r}^{\prime}\right)=\frac{i}{4} H_{0}^{(1)}\left(k\left|\vec{r}-\vec{r}^{\prime}\right|\right)
$$

and the unknown density function $Y(\vec{r})$ is the "effective" surface current

$$
Y(\vec{r})=H^{+}(\vec{r})-H^{-}(\vec{r}), \quad \vec{r} \in M .
$$

By using the boundary conditions (2), (3) and the property of the normal derivative of a double-layer potential, one arrives at the following integral equation:

$$
\begin{aligned}
\frac{i k}{Z_{0}} R(\vec{r}) Y(\vec{r}) & +\frac{\partial}{\partial n} \int_{M} Y\left(\vec{r}^{\prime}\right) \frac{\partial}{\partial n^{\prime}} G_{0}\left(\vec{r}, \vec{r}^{\prime}\right) d l^{\prime} \\
& =-\frac{\partial H^{i n}(\vec{r})}{\partial n}, \quad \vec{r} \in M .
\end{aligned}
$$

This is a singular IE with the kernel singularity of the Cauchy type, that makes a direct solution by a MoM-like algorithm dependent on the implementation. Equation (10) is valid for any open curve $M$ representing the contour of the reflector. Further, we shall follow [4] and [10] and restrict our analysis to parabolic reflectors which can be well approximated by circular ones (see [4, Fig. 2]). Then we can assume that $M$ is a circular arc of radius $a$, which is twice the focal distance of the paraboloid $F$ (Fig. 1). The aperture dimension $D$ is determined by the angle $2 \theta_{\text {ap }}$. Further, we extend the function $Y(\vec{r}) \equiv Y(a, \phi)$ beyond $M$ by setting its value to zero on the rest of the circle of radius $a$ and expand it in terms of the whole-period angular exponents as

$$
Y(\phi)=\frac{2 C}{i \pi k a} \sum_{n=-\infty}^{\infty} y_{n} e^{i n \phi} .
$$

Similar expansions are introduced for the kernel and the right-hand part of IE (10) that are the same as corresponding 


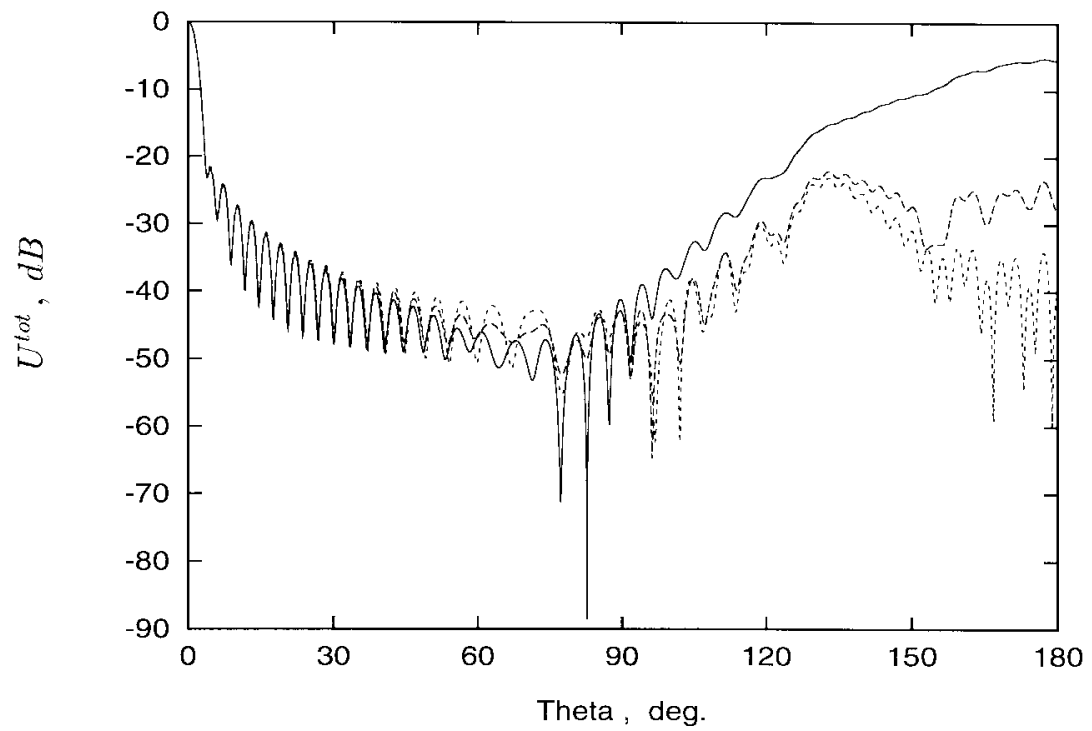

(a)

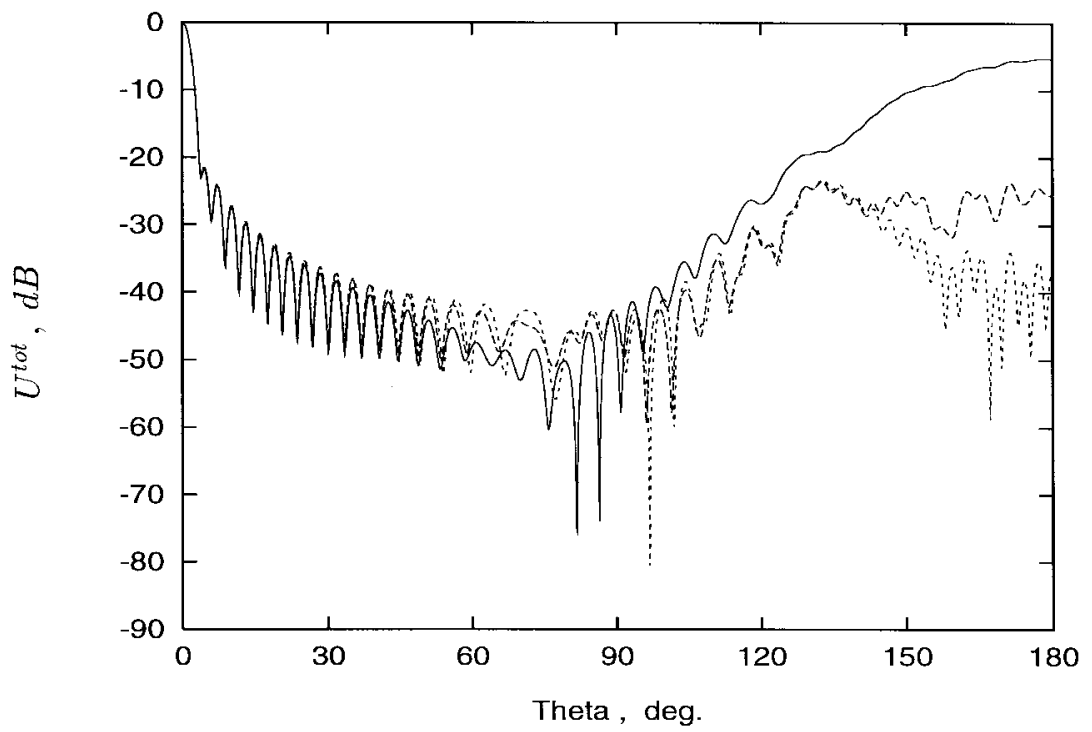

(b)

Fig. 2. Normalized far-field patterns of the H-polarized complex-point source feeding. (a) Lossy $(\operatorname{Im} R=0$ ). (b) Lossless $($ Re $R=0)$ uniformly resistive reflector. Feed parameter $k b=5$ corresponds to the $-9.5-\mathrm{dB}$ edge illumination. Reflector parameters $k a=183.7$ and $\theta_{a p}=20^{\circ}$ correspond to $D=20 \lambda, F / D=0.73$. The resistivity values are $0.01 Z_{0}$ (dotted curve), $0.1 Z_{0}$ (dashed curve), and $Z_{0}$ (solid curve).

expressions in PEC case [4]. Besides, we expand the resistivity function as

$$
R(\phi)=Z_{0} R_{0}\left(1+\sum_{n=-\infty, \neq 0}^{\infty} \rho_{n} e^{i n \phi}\right)
$$

and assume that the series (11) and (12) are uniformly convergent.

Substituting these expansions to the IE (10) and integrating from 0 to $2 \pi$ yields the following dual series equations:

$$
\begin{aligned}
\sum_{n=-\infty}^{\infty} y_{n}\left(J_{n}^{\prime} H_{n}^{\prime}+\frac{2 R_{0}}{\pi k a}\right) e^{i n \phi} & \\
& +\frac{2 R_{0}}{\pi k a} \sum_{n=-\infty}^{\infty} \sum_{p=-\infty, \neq 0}^{\infty} y_{n} \rho_{p} e^{i(n+p) \phi}
\end{aligned}
$$

$$
\begin{gathered}
=-\sum_{n=-\infty}^{\infty} J_{n}\left(k r_{s}\right) H_{n}^{\prime} e^{i n \phi}, \quad|\phi|<\theta_{a p} \\
\sum_{n=-\infty}^{\infty} y_{n} e^{i n \phi}=0, \quad \theta_{a p}<|\phi| \leq \pi .
\end{gathered}
$$

Here, $J_{n}=J_{n}(k a), H_{n}=H_{n}^{(1)}(k a)$ and the prime denotes differentiation with respect to the argument. The series on the right-hand part of (13) is uniformly convergent for all $\left|r_{s}\right|<a$, where for the front-fed reflector

$$
r_{s}=r_{0}+i b
$$

If the feed is placed in the geometrical focus of reflector, $r_{0}=a / 2$.

The expansion coefficients $\rho_{n}$ for the resistivity should decay with larger $n$, but their explicit large-index behavior is 
of secondary importance as the main contribution to the weight function in (13) is given by the term $J_{n}^{\prime} H_{n}^{\prime} \sim i|n| /\left(\pi k^{2} a^{2}\right)$. The latter is the same as in PEC case studied in [4]. Hence, the dual series equations (13), (14) can be partially inverted by using analytical solution of the so-called Riemann-Hilbert problem [11] in the form given in [12]. As a result, we come to the following infinite matrix equation:

$$
\left(I-A^{H}\right) Y=B^{H}
$$

where

$$
\begin{aligned}
I_{m n}= & \delta_{m n} \\
A_{m n}^{H}= & K_{n}\left(k a, R_{0}\right) T_{m n}\left(\theta_{a p}\right) \\
& +i 2 \pi k a R_{0} \sum_{p=-\infty, \neq 0}^{\infty} \rho_{p} T_{m, n+p}\left(\theta_{a p}\right) \\
K_{n}\left(k a, R_{0}\right)= & i 2 k a R_{0}+|n|+i \pi(k a)^{2} J_{n}^{\prime} H_{n}^{\prime} \\
B_{m}^{H}= & i \pi(k a)^{2} \sum_{n=-\infty}^{\infty} J_{n}\left(k r_{s}\right) H_{n}^{\prime} T_{m n}\left(\theta_{a p}\right) .
\end{aligned}
$$

The angular coefficients $T_{m n}\left(\theta_{a p}\right)$ are the same as for the $\mathrm{H}$ scattering by a PEC reflector [4] and are given in the Appendix. The large-index behavior of the matrix elements is estimated as

$$
A_{m n}^{H} \sim O\left[(|m-n|+1)^{-1}|m n|^{-1 / 2}\right]
$$

which enables one to prove the Fredholm nature of (16) and the needed edge behavior. Note that the second term in (17) vanishes provided that the resistivity is a constant on the contour $M$, thus reducing the solution to the case considered in [10]. Also worth noting is that the terms proportional to $R_{0}$ play the role of a perturbation to the PEC equation in (16) for any $k$ (compare with [4], [11], and [12]). This is not a surprise as (16) is equivalent to IE (10), which contains $k R$-proportional term also as a nonsingular perturbation to the IE of the PEC case. Generally, the matrix elements $A_{m n}^{H}$ vanish if $k a=0$, which points out to the fact that deriving (16) is equivalent to inverting the static part of the dual series equations. When making computations, it is recommended to split the field and (16) into symmetric and antisymmetric parts, thus reducing by half the order of the matrices to be inverted (see [4]). As was verified in [10], for 3 -digit accuracy it is enough to truncate the matrix size to $N_{t r}=\left(1+\left|2 R_{0}\right|^{1 / 2}\right) k a+5$.

\section{Basic Equations for the E-Polarization CASE}

In this case, we seek the $z$-component scattered electric field function as a single-layer potential

$$
E^{s c}(\vec{r})=\int_{M} X\left(\vec{r}^{\prime}\right) G_{0}\left(\vec{r}, \vec{r}^{\prime}\right) d l^{\prime}
$$

where the unknown "effective" current density function is defined as

$$
X(\vec{r})=-\left[\frac{\partial}{\partial n} E^{+}(\vec{r})-\frac{\partial}{\partial n} E^{-}(\vec{r})\right], \quad \vec{r} \in M .
$$

By using the boundary conditions (2) and (3) and the property of the single-layer potential when crossing the layer, one obtains the following electric field IE:

$$
\begin{gathered}
-X(\vec{r}) R(\vec{r})+i k Z_{0} \int_{M} X\left(\vec{r}^{\prime}\right) G_{0}\left(\vec{r}, \vec{r}^{\prime}\right) d l^{\prime} \\
=-i k Z_{0} E^{i n}(\vec{r}), \quad \vec{r} \in M .
\end{gathered}
$$

Note that if $R$ is not identically zero, (23) is already a regularized Fredholm second-kind IE as its kernel has a logarithmic (i.e., integrable) singularity. However, unlike the previous $\mathrm{H}$-pol case, here the $R$-dependent term plays the role of a nonsingular perturbation of the PEC IE only provided that $k \neq 0$. It means that (23) does not permit a continuous transition to the static case of a PEC reflector. Practically speaking, the quasi-static behavior of a resistive reflector can be much different from PEC approximation even if $R$ is very small. The regularized nature of (23) guarantees that a simple MoM-like algorithm to solve it will be stable and convergent to the exact solution as the number of expansion functions get large.

Further, we assume (as in the previous section) that the reflector can be approximated by a circular one and extend $X(\vec{r})$ by identical zero off $M$ over the circular contour. Then we expand the current and the resistivity in terms of the angular exponents as

$$
\begin{aligned}
X(\vec{r}) & \equiv X(\phi)=\frac{2 C}{i \pi a} \sum_{n=-\infty}^{\infty} x_{n} e^{i n \phi} \\
\frac{1}{R(\vec{r})} & =\frac{1}{Z_{0} R_{0}}\left(1+\sum_{p=-\infty, \neq 0}^{\infty} \gamma_{p} e^{i p \phi}\right)
\end{aligned}
$$

assuming that $R_{0} \neq 0$.

Expanding also the kernel and the right-hand part of (23) in the same manner as in PEC case [4] and using the orthogonality of the exponentials leads to the following dual series equations:

$$
\begin{aligned}
& \sum_{n=-\infty}^{\infty} x_{n}\left(\frac{2 R_{0}}{\pi k a}+J_{n} H_{n}\right) e^{i n \phi} \\
& +\sum_{n=-\infty}^{\infty} \sum_{p=-\infty, \neq 0}^{\infty} x_{n} \gamma_{p} J_{n} H_{n} e^{i(n+p) \phi} \\
& =-\sum_{n=-\infty}^{\infty}\left(J_{n}\left(k r_{s}\right) H_{n} e^{i n \phi}\right. \\
& \left.+\sum_{p=-\infty, \neq 0}^{\infty} \gamma_{p} J_{n}\left(k r_{s}\right) H_{n} e^{i(n+p) \phi}\right) \\
& \left.|\phi|<\theta_{a p}\right) \\
& \sum_{n=-\infty}^{\infty} x_{n} e^{i n \phi}=0, \quad \theta_{a p}<|\phi| \leq \pi
\end{aligned}
$$

Unlike the H-pol case, (26) and (27) can be partially inverted for all $R_{0} \neq 0$ by using a simple inverse Fourier transform. Here we follow [10] and come to the following regularized infinite matrix equation:

$$
\left(\boldsymbol{I}-A^{E}\right) \boldsymbol{X}^{\prime}=\boldsymbol{B}^{E}
$$




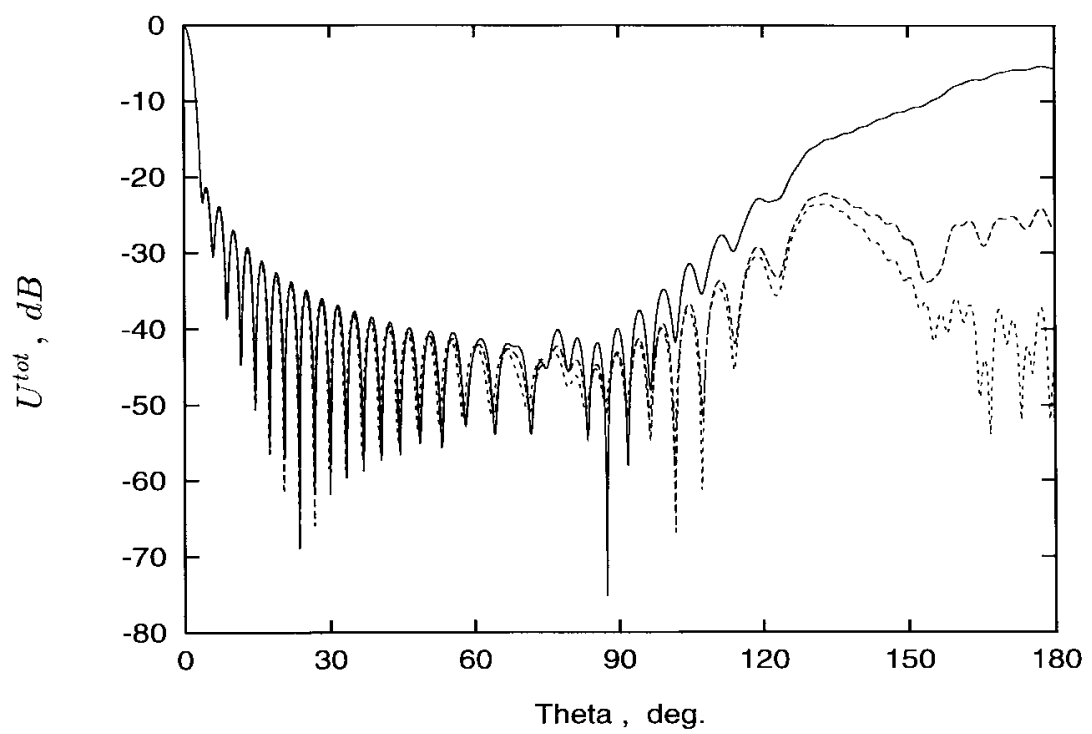

(a)

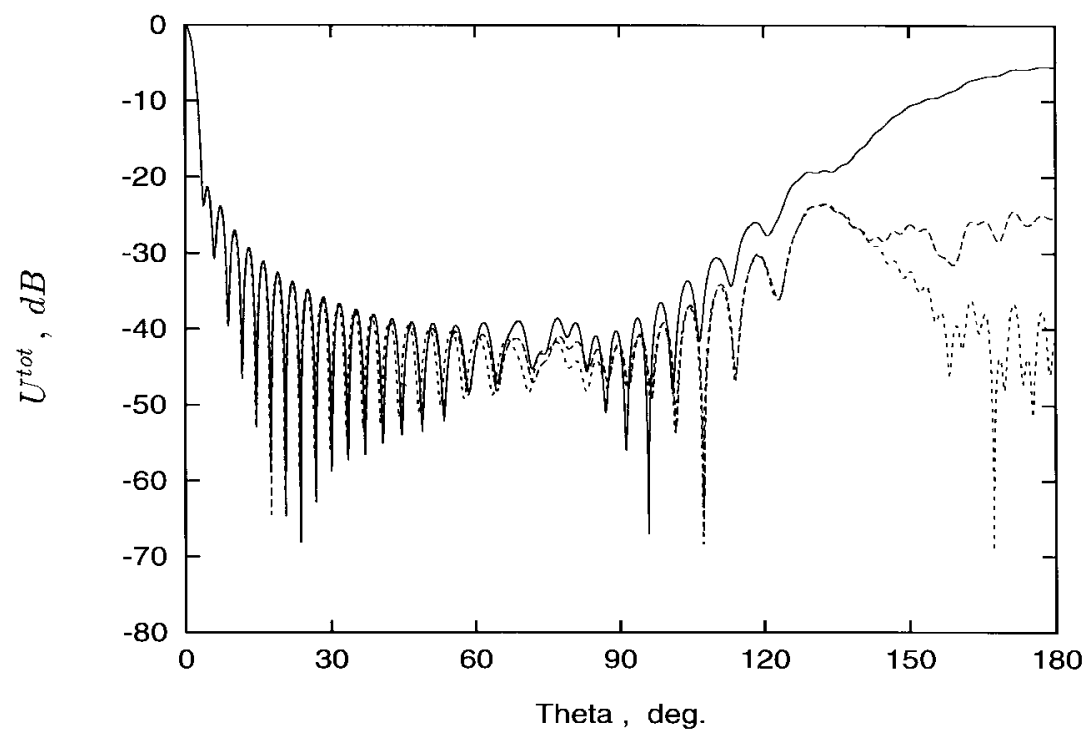

(b)

Fig. 3. The same as in Fig. 2, but for the E-polarization case.

where

$$
\begin{aligned}
I_{m n} & =\delta_{m n}, \quad x_{n}^{\prime}=x_{n}(|n|+1)^{-1 / 2} \\
A_{m n}^{E} & =-\frac{k a}{2 R_{0}} J_{n} H_{n} Q_{m n}\left(\frac{|n|+1}{|m|+1}\right)^{1 / 2} \\
Q_{m n} & =S_{m n}\left(\theta_{a p}\right)+\sum_{p=-\infty, \neq 0}^{\infty} \gamma_{p} S_{m, n+p}\left(\theta_{a p}\right) \\
B_{m}^{E} & =\frac{-k a}{2 R_{0}(|m|+1)^{1 / 2}} \sum_{n=-\infty}^{\infty} J_{n}\left(k r_{s}\right) H_{n} Q_{m n} .
\end{aligned}
$$

The angular coefficients $S_{m n}$ are given in the Appendix. Note that the matrix elements $A_{m n}^{E}$ vanish if $k a=0$. It means that reducing the dual-series equations to (28) is equivalent to inverting their static part. However, a continuous transition to the PEC static case is not possible. The large-index behavior of the matrix elements $A_{m n}^{E}$ is easily verified to be the same as in H-case given by (20). According to [10], for three-digit accuracy it is enough to take $N_{t r}=\left(1+\left|2 R_{0}\right|^{-1 / 2}\right) k a+$ 5 equations provided that the symmetry splitting has been utilized.

\section{NUMERICAL Results}

\section{A. Uniformly Resistive Reflector-H- and E-Polarizations}

Here we would like to present and discuss some results of numerical modeling of a uniformly resistive reflector. It means that we take $\rho_{n}=0$ and $\gamma_{n}=0$ for all $n \neq 0$. The geometry of the reflector is determined by the parameters $k a=183.7$ and $\theta_{a p}=20^{\circ}$, which is equivalent to a parabolic cylinder of $D=20 \lambda$ and $F / D=0.75$, with an error less than $\lambda / 16$. A feed characterized by $k b=5$ is placed in the geometrical focus $\left(r_{0}=a / 2\right)$ resulting in an edge illumination level of $-9.5 \mathrm{~dB}$ (Fig. 1). 


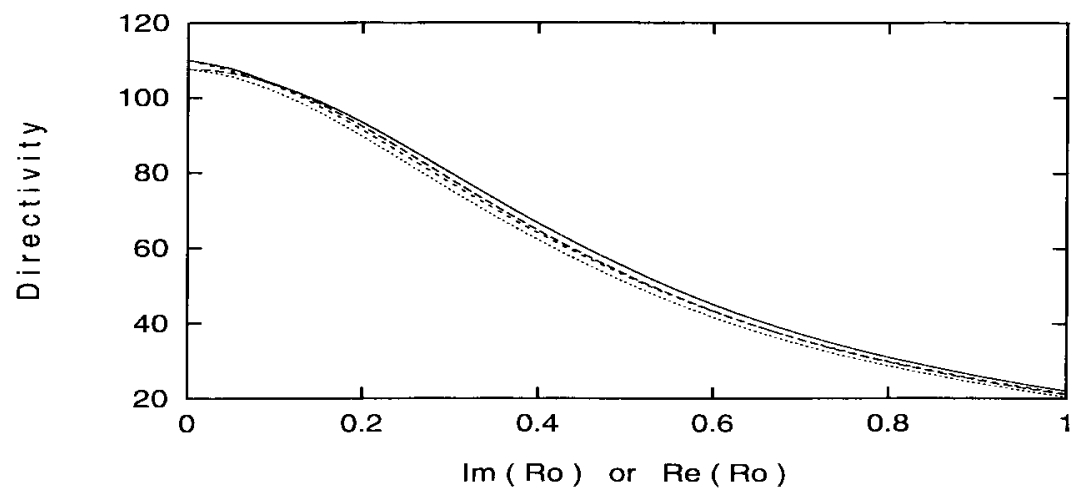

(a)

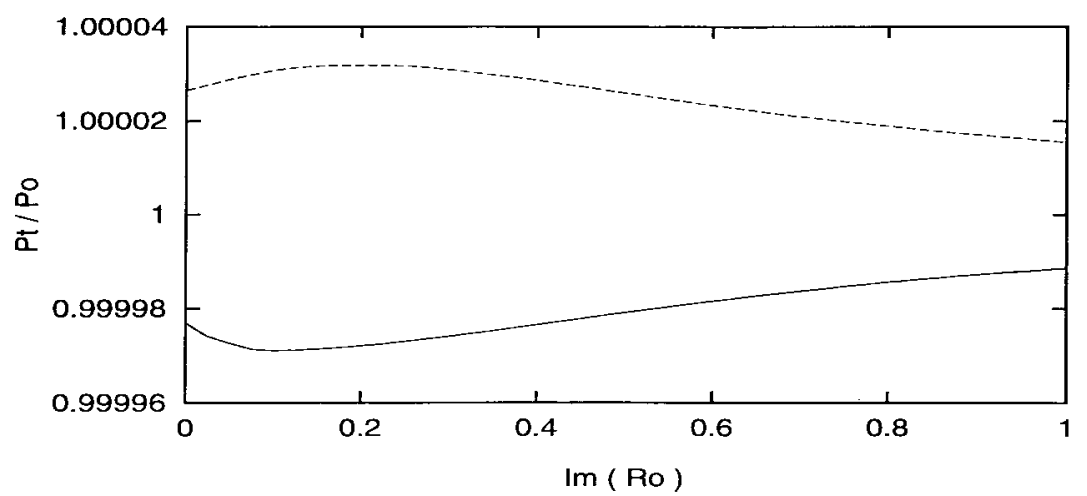

(b)

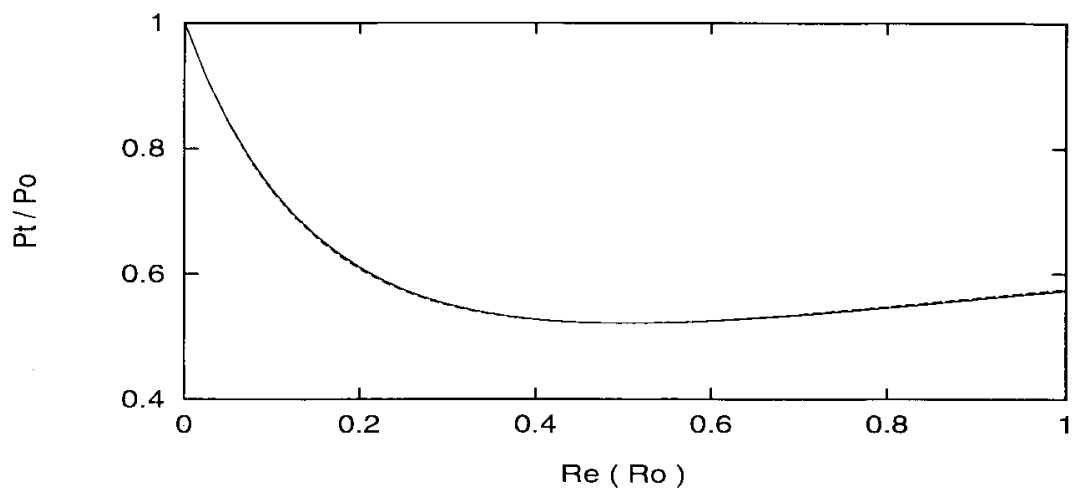

(c)

Fig. 4. (a) Directivity. (b) and (c) Normalized total radiated power as a function of resistivity. E-polarization: (a) and (b) solid curves for lossless reflector; (a) short-dashed and (c) solid curves for lossy one. H-polarization: (a) long-dashed and (b) dashed curves for lossless reflector; (a) dotted and (c) dashed curves for lossy one.

The particular operating characteristics that we analyze are the far-field radiation pattern, the normalized total radiated power and the directivity. These parameters can be easily computed after solving the matrix equation (16) or (28) for the surface-current expansion coefficients. The corresponding formulas are the series given in [4] by (21), (23), and (24).

In Figs. 2 and 3, one can see the effect of nonzero resistivity on the far-field patterns of such a reflector for two alternative polarizations. Both lossy $(\operatorname{Im} R=0)$ and lossless $(\operatorname{Re} R=0)$ reflector patterns are presented. The larger the resistivity, the greater is the feed-field leakage through the reflector due to the partial transparency. If the relative resistivity $R_{0}=R / Z_{0}$ is of the order of $10^{-3}$ or less, the patterns graphically coincide with the PEC ones. H-pol and E-pol case patterns of such a 20- $\lambda$ reflector are similar except for the rear sidelobes, which are a bit higher in the $\mathrm{H}$-case due to stronger edge scattering. The greatest sidelobe peak, which occurs at about $130^{\circ}$ is due to spillover. A lossy (thinner-than-skindepth metal) screen forms a pattern which is very similar to that formed by a lossless (thin dielectric) one of the same absolute value of $R$.

It is worth noting that the computational algorithm based on the MoR approach is very fast. In the H-pol case, for example, when the matrix truncation number increases with increasing $R$, computation of the highest resistivity curve in Fig. $2\left(R=Z_{0}\right)$ for three-digit accuracy requires $N_{t r}=448$, but it takes only $69 \mathrm{~s}$ of CPU time on a SUN SPARCStation 


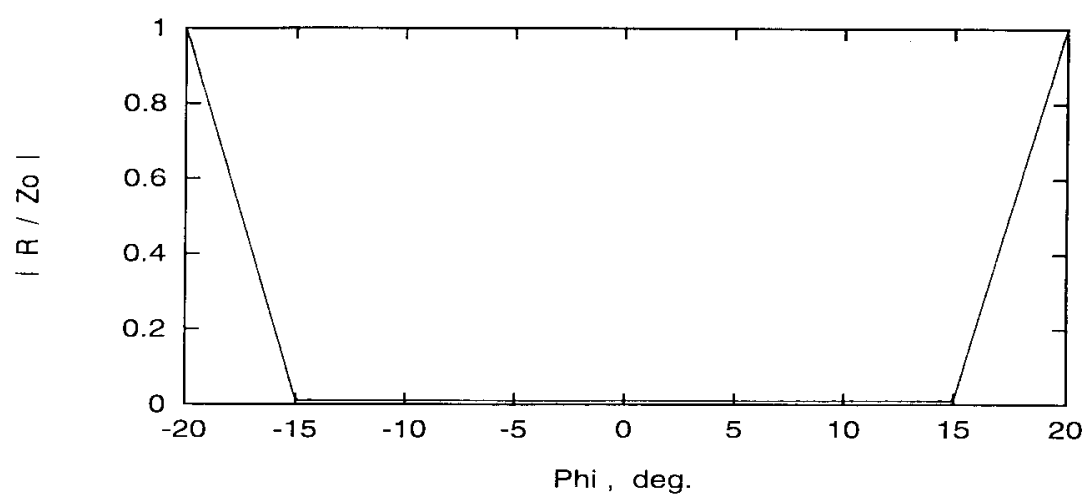

(a)

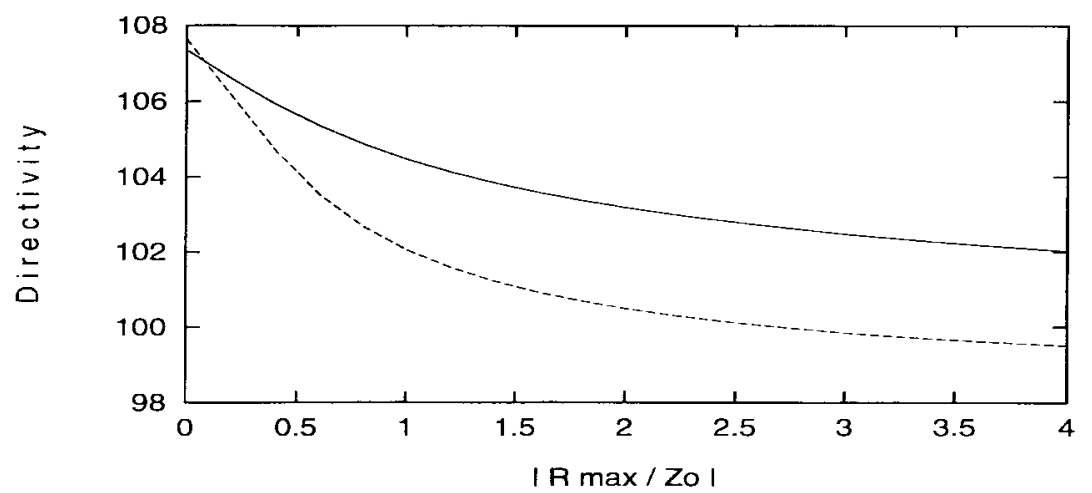

(b)

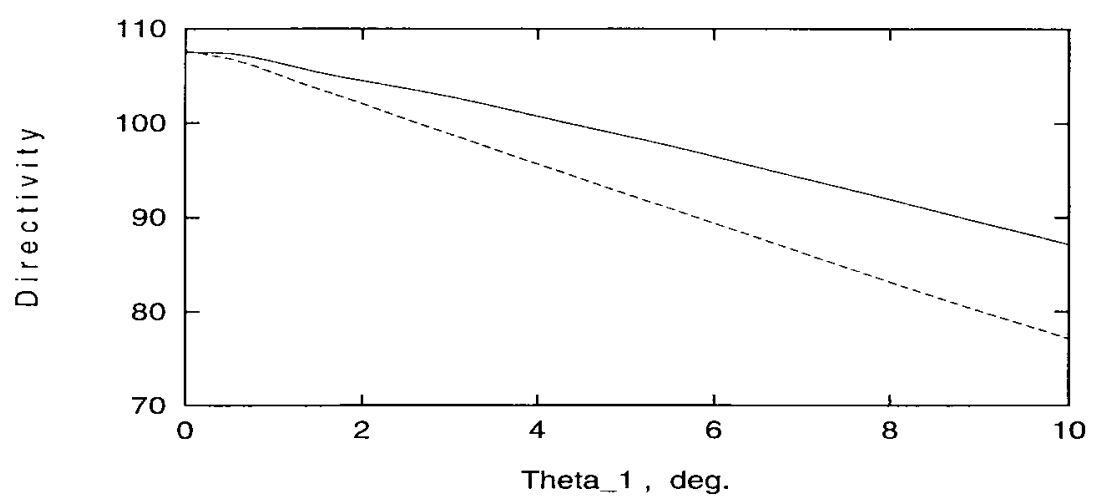

(c)

Fig. 5. (a) Resistivity of the edge-loaded reflector. (b) and (c) Directivity as a function of $\left|R_{\max }\right|\left(\theta_{1}=2^{\circ}\right)$ and of angular width $\theta_{1}\left(\left|R_{\max }\right|=Z_{0}\right)$ of a linear-varied edge loading, for lossy (solid curve) and lossless (dashed curve) reflector, H-polarization. $\left|R_{\min }\right|=0.01 Z_{0}$ in all cases.

20 at $60 \mathrm{MHz}$, with memory consumption of about $8 \mathrm{Mb}$ for double-precision FORTRAN code under the SUN OS 4.1.4 UNIX operating system.

Fig. 4 demonstrates the effect of changing the value of resistivity of a lossless and lossy reflector on the antenna directivity and the normalized total radiated power. Note that the radiated power decays very rapidly with the resistivity of a lossy reflector, at the expense of the growth of the power absorbed in the reflector material. This results in the drop in the overall gain of antenna.

\section{B. Edge-Loaded Reflector-H-Polarization}

When studying the variable-resistivity reflector, we assumed that the total aperture of the reflector was the same $20-\lambda$ value as before, but the resistivity function was as given in Fig. 5(a). The linear rise, within the edge area of width $d_{1}$ from the constant value in the central part $R e R=0.01 Z_{0}$ (a good conductivity, close to PEC case according to [10]) to the edge value $\mathrm{Re} R=\mathrm{Z}_{0}$ (highly transparent surface), was approximated by 10 to 20 terms of the series (12) depending on the loading angular width $\theta_{1}$. In our notations, $d_{1} / \lambda=(k a / \pi) \sin \left(\theta_{1} / 2\right)$ (see Fig. 1). An H-polarized feed having an aperture of $k b=5$ resulted in the edge illumination level of $-9.5 \mathrm{~dB}$.

In Figs. 6 and 7, the far-field radiation patterns are presented for two different values of $\theta_{1}$. In Fig. 6, the effect of the resistive edge loading is clearly seen as lowering the level of sidelobes in the forward half-space. The difference can 


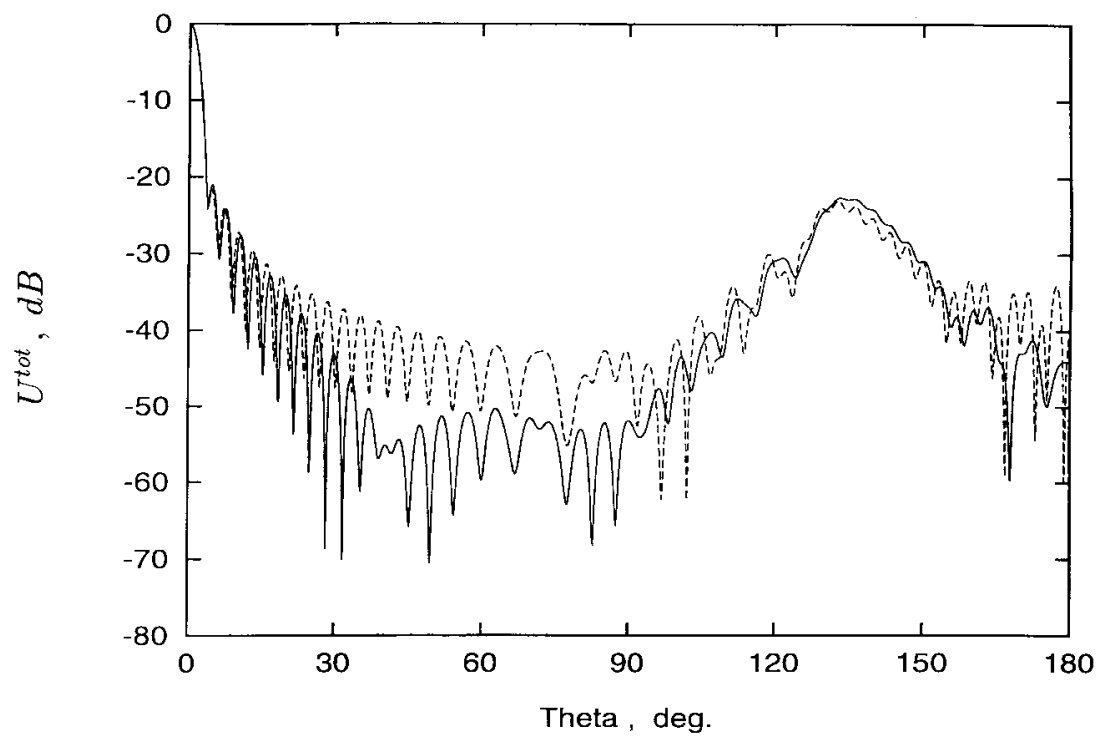

(a)

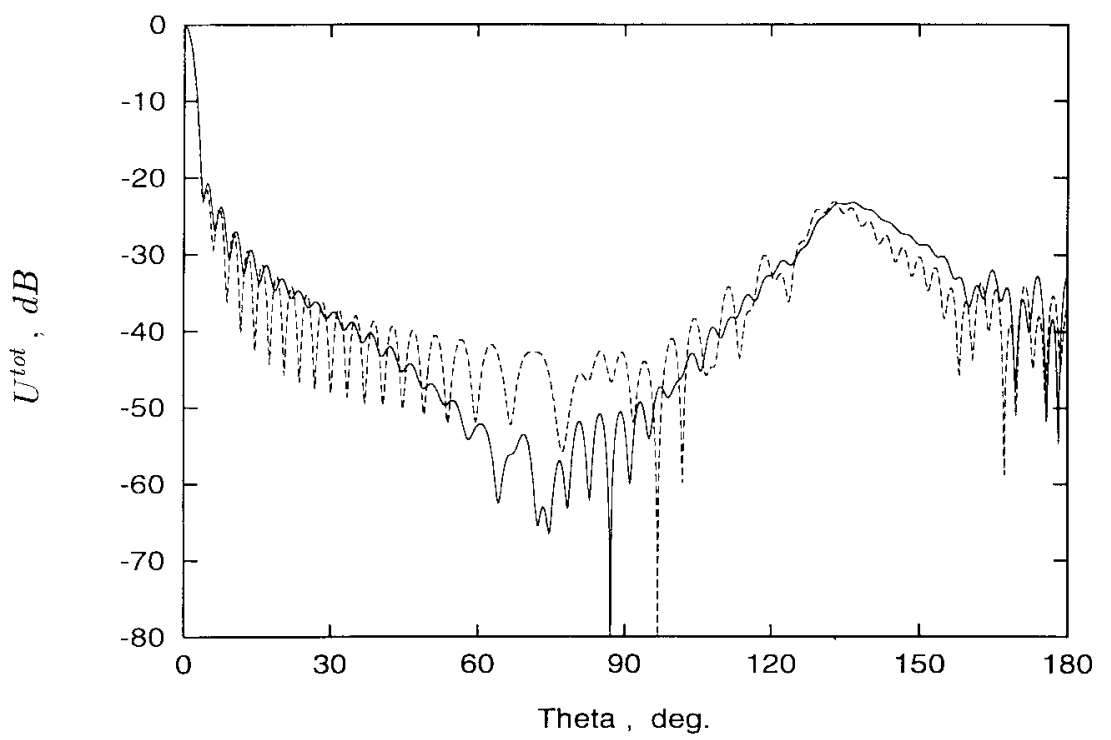

(b)

Fig. 6. Far-field pattern of (a) lossy and (b) lossless reflector with a linear-varied resistive edge loading (solid curve) compared with a uniformly-resistive reflector of $|R|=0.01 Z_{0}$ (dashed curve), for H-polarization case. Loading width $\theta_{1}=2^{\circ},\left|R_{\max }\right|=Z_{0},\left|R_{\min }\right|=0.01 Z_{0}$. Other parameters as in Fig. 2 .

reach some $10 \mathrm{~dB}$ depending on the width of the loaded strip. However, the latter should not be too wide, as the overall directivity is always undermined by a greater fraction of the feed field getting through (see Fig. 7). The directivity loss is greater for a lossless edge loading than for a lossy one, which is evident from Fig. 5(b) and (c). The value of the total radiated power (not shown) is hardly affected by such a loading.

\section{CONCLUSIONS}

We have demonstrated that MoR analysis based on the analytical inversion of the static part of electric-field IE can be generalized to cover a thin variable-resistivity reflector. To this end, we reduce the IE to the dual-series equations and further extract the static part out of the corresponding operator. The latter is essentially different for the $\mathrm{H}$ and $\mathrm{E}$ polarizations. In the $\mathrm{H}$ case, it is the same as in the PEC case, so the method of its inversion is based on the solution of the so-called Riemann-Hilbert problem. The effect of finite resistivity is seen as an additional term proportional to the product $k R$ and playing the role of a perturbation to the PEC reflector scattering. In the $E$ case, the presence of finite resistivity changes the IE and the dual-series equations in a fundamental manner, so that the static part is completely different. In fact, it is now simpler than in the PEC case and can be inverted by using the inverse Fourier transform. Both polarization solutions are valid for nonuniform variableresistivity reflectors, although with a uniform resistivity the needed matrix size may be smaller.

The operating characteristics of 2-D reflectors were analyzed by using a complex-point source model of the primary feed. This model has already proved its advantages of accuracy and simple implementation in the PEC case [4]. Here, we 


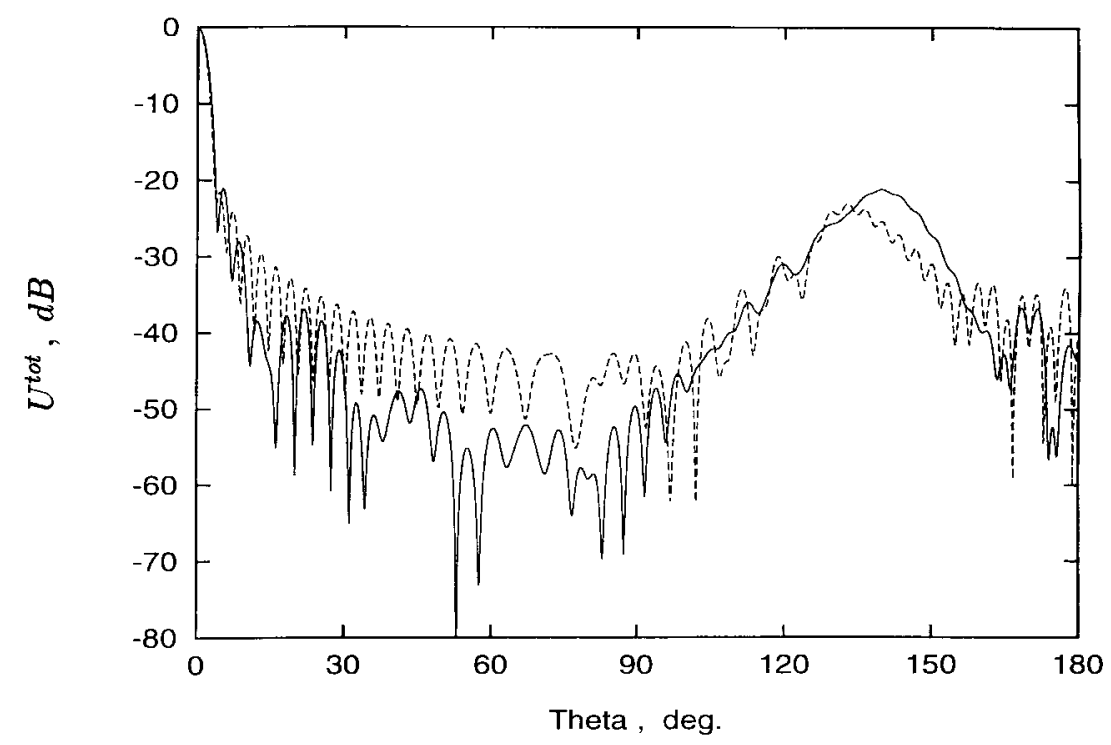

(a)

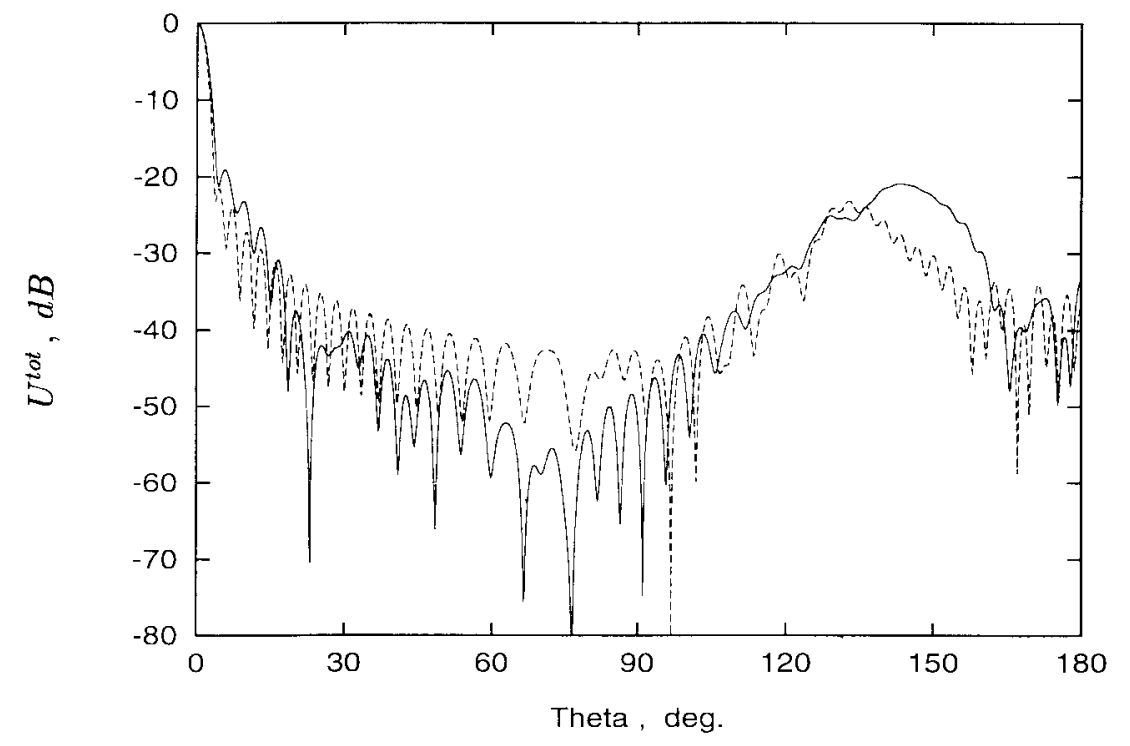

(b)

Fig. 7. The same as in Fig. 6, but for the loading width $\theta_{1}=6^{\circ}$.

applied it to the resistive reflector. Our aim was to study the effect of a uniform and a nonuniform resistivity on the far-field radiation pattern and overall parameters such as normalized total radiated power and directivity.

A uniform resistivity results in a partial transparency of the reflector. Hence, the rear-side radiation becomes higher and the directivity lower, but the main beam and near sidelobes remain surprisingly the same.

The most interesting results have been obtained for the edgeloaded reflector. A careful playing with both the width of the higher resistive edge zone of the reflector and the character of resistivity variation in this zone results in a well-observable lowering of the sidelobe level. This is especially significant in the forward half-space. A small directivity attenuation is, however, inescapable due to the partial transparency of the edge area. Here, a lossy loading shows a better performance than a lossless one. Thus, intuitive considerations about a positive effect of the edge loading have been supported by the accurate numerical analysis of the full-wave integral equations.

\section{APPENDIX}

Consider the dual-series equations as follows:

$$
\begin{aligned}
\sum_{n=-\infty}^{\infty} x_{n}|n| e^{i n \phi} & =\sum f_{n} e^{i n \phi}, \quad|\phi|<\theta_{a p} \\
\sum_{n=-\infty}^{\infty} x_{n} e^{i n \phi} & =0, \quad \theta_{a p}<|\phi| \leq \pi
\end{aligned}
$$

where $x_{n}$ are the unknowns and $f_{n}$ are assumed known values.

According to [12] the analytical solution to the above equations can be written as

$$
x_{m}=\sum_{n=-\infty}^{\infty} f_{n} T_{m n}\left(\theta_{a p}\right), \quad m=0, \pm 1, \cdots
$$


where

$$
\begin{aligned}
T_{m n}\left(\theta_{a p}\right) & =\frac{\left[P_{m-1}(u) P_{n}(u)-P_{m}(u) P_{n-1}(u)\right]}{2(m-n)}, \quad m \neq n \\
T_{n n}\left(\theta_{a p}\right) & =\frac{1}{2|n|} \sum_{s=0}^{|n|} q_{|n|-s}(u) P_{|n|-s-1}(u), \quad n \neq 0 \\
T_{00}\left(\theta_{a p}\right) & =-\ln \frac{1+u}{2}
\end{aligned}
$$

with $u=\cos \theta_{a p}, q_{0}=1, q_{1}=-u, \cdots, q_{s}=P_{s}(u)-$ $2 u P_{s-1}(u)+P_{s-2}(u)$, and $P_{s}(u)$ being the Legendre polynomials.

Consider further the following series equations:

$$
\sum_{n=-\infty}^{\infty} x_{n} e^{i n \phi}= \begin{cases}\pi \sum_{n=-\infty}^{\infty} f_{n} e^{i n \phi}, & |\phi|<\theta_{a p} \\ 0, & \theta_{a p}<|\phi| \leq \pi\end{cases}
$$

where $x_{n}$ are the unknowns and $f_{n}$ are assumed known values.

By multiplying both sides of (35) with $e^{-i m \phi}$ and integrating from 0 to $2 \pi$, the analytical solution is obtained as

$$
x_{m}=\sum_{n=-\infty}^{\infty} f_{n} S_{m n}\left(\theta_{a p}\right), \quad m=0, \pm 1, \cdots
$$

where

$$
\begin{aligned}
S_{m n}\left(\theta_{a p}\right) & =\frac{\sin \left[(n-m) \theta_{a p}\right]}{(n-m)}, \quad m \neq n \\
S_{n n}\left(\theta_{a p}\right) & =\theta_{a p} .
\end{aligned}
$$

\section{ACKNOWLEDGMENT}

The authors would like to thank the reviewers for improving the manuscript.

\section{REFERENCES}

[1] H. Yokoi and H. Fukumuru, "Low sidelobes of paraboloidal antennas with microwave absorbers," Electromagn. Commun. Jpn., vol. 54-B, no. 11, pp. 34-49, 1971.

[2] O. Bucci and G. Franceschetti, "Rim loaded reflector antennas," IEEE Trans. Antennas Propagat., vol. AP-28, pp. 297-304, May 1980.

[3] O. Bucci, G. Di Massa, and C. Savarese, "Control of reflector antennas performance by rim loading," IEEE Trans. Antennas Propagat., vol. AP-29, pp. 773-779, Sept. 1981.

[4] T. Oguzer, A. I. Nosich, and A. Altintaş, "Accurate simulation of reflector antennas by complex source-Dual series approach," IEEE Trans. Antennas Propagat., vol. 43, pp. 793-801, Aug. 1995.

[5] T. B. A. Senior, "Some problems involving imperfect half-planes," P. L. E. Uslenghi, Ed., Electromagnetic Scattering. Orlando, FL: Academic, 1978, pp. 185-219.

[6] G. Bouchittè and R. Petit, "On the concepts of a perfectly conducting material and of a perfectly conducting and infinitely thin screen," Radio Sci., vol. 24, no. 1, pp. 13-26, 1989.

[7] E. Bleszynski, M. Bleszynski, and T. Jaroszewicz, "Surface-integral equations for electromagnetic scattering from impenetrable and penetrable sheets," IEEE Trans. Antennas Propagat. Mag., vol. 35, pp. 14-25, June 1993.

[8] T. B. A. Senior and J. Volakis, Approximate Boundary Conditions in Electromagnetics. Piscataway, NJ: IEEE Publ., 1995.

[9] E. V. Jull and G. A. Suedan, "Beam diffraction by planar and parabolic reflectors," IEEE Trans. Antennas Propagat., vol. 39, pp. 521-527, Apr. 1991.

[10] A. I. Nosich, Y. Okuno, and T. Shiraishi, "Scattering and absorption of E and H-polarized plane waves by a circularly curved resistive strip," Radio Sci., vol. 31, no. 6, pp. 1733-1742, 1996.
[11] R. W. Ziolkowski, "N-series problems and the coupling of the electromagnetic waves to apertures: Riemann-Hilbert approach," SIAM J. Appl. Math., vol. 16, pp. 358-378, 1985.

[12] A. I. Nosich, "Green's function-Dual series equations in wave scattering from resonant scatterers," in M. Hashimoto, M. Idemen, and O. A. Tretyakov, Eds., Analytical and Numerical Methods in Electromagnetic Wave Theory. Tokyo, Japan: Science House, pp. 419-469, 1993.

Alexander I. Nosich (M'94-SM'95) was born in Kharkov, Ukraine, in 1953. He received the M.S., Ph.D., and D.Sc. degrees in radio physics from Kharkov University, Ukraine, in 1975, 1979, and 1990, respectively.

Since 1978, he has been with the Electronics Department of the Institute of Radiophysics and Electronics (IRE) of the Ukrainian Academy of Sciences, Kharkov, as a Junior and Senior Research Scientist. In 1992 he was with the Electrical and Electronics Engineering Department, Bilkent University, Ankara, Turkey, as a Visiting Professor through a fellowship of the Turkish Scientific and Technical Research Council (TUBITAK). From 1992 to 1994 he was with the Electrical Engineering and Computer Science Department, Kumamoto University, Kumamoto, Japan, as a Guest Scientist an Associate Professor, through a fellowship of the Matsumae International Foundation. In 1996 and 1997 he was a Visiting Professor with the Universite de Rennes 1, France. Currently, he is a Leading Scientist at the Institute of Radiophysics and Electronics (IRE), Kharkov. His research interests include free-space and open waveguide scattering, complex-mode behavior, radar cross-section modifying, and antenna simulation.

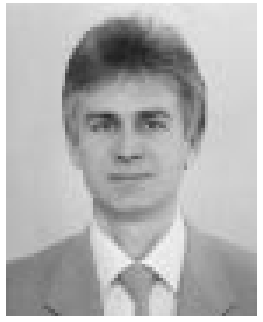

Vladimir B. Yurchenko was born in 1957 in Kharkov, Ukraine. He received the M.S. degree (with honors) from the Physics and Technology Department, Kharkov Polytechnic Institute, Kharkov, Ukraine, the Ph.D. degree (solid state physics) from the Kharkov Polytechnic Institute, and the D.Sc. degree from the Institute of Radiophysics and Electronics (IRE), Ukrainian Academy of Sciences, Kharkov, in 1980, 1984, and 1994, respectively.

From 1984 to 1995 , he was with Department of Materials for Electronics and Solar Cells, Kharkov Polytechnic Institute, Ukraine, as a Research Scientist and an Associate Professor. His research interests there included the theory of hot-electron transport, photovoltaics, and electrical instability in semiconductors. Since 1995 he has been with the Institute of Radiophysics and Electronics (IRE) as a Senior Research Scientist in the Department of Nonlinear Dynamics of Electronic Systems. Since 1996, he has been a member of the visiting faculty at the Department of Electrical and Electronics Engineering, Bilkent University, Ankara, Turkey. His current interests include computer simulation of electron transport in nanostructures, dynamical chaos in electronic devices, and wave scattering in electromagnetic systems.

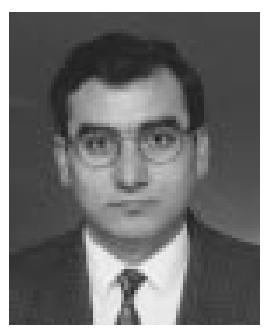

Ayhan Altintaş (S'82-M'86-SM'93) was born on March 29, 1958, in Yozgat, Turkey. He received the B.S. and M.S. degrees from the Middle East Technical University (METU), Ankara, Turkey, in 1979 and 1981, respectively, and the Ph.D. degree from The Ohio State University, Columbus, $\mathrm{OH}$, in 1986, all in electrical engineering.

From 1981 to 1987 , he was with the ElectroScience Laboratory of The Ohio State University. Currently, he is a Professor at the Department of Electrical and Electronics Engineering, Bilkent University, Ankara, Turkey. His research interests include electromagnetic radiation and scattering, microwaves, fiber optics, and integrated optics.

Dr. Altintaş is the recipient of the ElectroScience Laboratory Outstanding Dissertation Award of 1986, the IEEE 1991 Outstanding Student Branch Counselor Award, the 1991 Research Award of the Professor Mustafa N. Parlar Foundation of METU, and the 1996 Young Scientist Award of TUBITAK. He is a member of Sigma Xi and Phi Kappa Phi. 